\title{
Subjective contours produced purely by dynamic occlusion of sparse-points array
}

\author{
TREVOR HINE \\ Massachusetts Institute of Technology, Cambridge, Massachusetts
}

\author{
(Charles W. Eriksen, Sponsor)
}

\begin{abstract}
In this paper, I describe the formation of a classic subjective contour by the occlusion of moving points of light having very sparse spatial distribution. In move and flash conditions, subjects determined what contour circumscribed the display region in which the points of light appeared. Subjects felt that the subjective contours were sharp and of distinct shape in the move condition if there were at least six impacts with the edges per second. No occluding subjective form was evident in the flash condition, although the delineation of an edge was more cogent than in the move condition.
\end{abstract}

Recently, researchers (Bradley \& Lee, 1982; Kellman $\&$ Cohen, 1984) have demonstrated that subjective contours can be produced by moving the inducing elements of the classic Kanisza (1976) figure. The strength of such subjective contours is reduced and their form becomes indistinct upon cessation of movement of the inducing elements. In addition, Klymenko and Weisstein (1981) showed that a rotating outline projection of a rectangular solid object produces a subjective contour across an empty region where an edge would normally have been located. However, the moving displays of these researchers were not true demonstrations of the formation of classic subjective contours purely by dynamic occlusion. In each case, virtually all the static frames of the displays contained edges and/or lines (standard inducing elements), which, by themselves and without movement, suggested a figural incompletion and a subjective contour (Coren, 1972). Movement simply enhanced the percept of a contour.

In this note, I describe the formation of a classic subjective contour purely by the occlusion of moving points of light that are of very sparse spatial distribution. There were no visible lines or edges for any static frame of the moving display. Rather, the subjective contour required time to form through spatiotemporal integration by the visual system. Because there were no visible lines or edges in the static frames, my display is distinguished from the dynamic occlusion demonstration of Kaplan (1969), in which an edge is immediately visible upon relative motion in a densely textured field, and also from the recent

I thank Andrew Parker, John Martin, and Brian McKeon for software development, and Eric Saund for initial discussions of the problem. Cath Graeber helped prepare the manuscript, and Graeme Cole and Michael Cook suggested improvements. The work was completed within the psychophysics lab at the Center for Biological Information Processing, Massachusetts Institute of Technology, Cambridge, MA.

Address reprint requests to the author's present address: Department of Applied Mathematics, Research School of Physical Sciences, Australian National University, GPO Box 4, Canberra, Australian Capital Territory 2601, Australia. demonstration of Prazdny (1986), in which the inducing elements themselves are defined by spatiotemporal correlation, rather than by brightness differences.

Finally, I conducted a small experiment that showed that the localization of the subjective contour is best when the paths of the points of light remain invisible and we simply flash the points at those moments when they are being occluded by the contour.

\section{METHOD}

\section{Stimuli and Apparatus}

Visual displays were produced by a microcomputer graphics system on a Tektronix 608 oscilloscope (CRT) with P-31 phosphor. This phosphor has a fast initial decay (in microseconds) but a sluggish secondary decay that begins at a luminance of about $1 \%$ of the original exposure. In a method similar to that of Bridgeman and Mayer (1983), I eliminated the effects of this secondary emission by diffusely illuminating the CRT screen so that the stimuli were barely visible to the observers. Thus the reduced contrast kept the secondary emission below threshold. The visual display consisted of either moving or flashed points of light, each point subtending $1.3^{\prime}$ of visual arc, within a rectangular region $\left(5^{\circ}\right.$ high $\times 6.25^{\circ}$ wide) on the CRT. In a well-lighted room and at a viewing distance of $40 \mathrm{~cm}$, subjects fixated a spot that was $0.4^{\circ}$ in diameter and situated in the center of the right-hand edge of the display region.

The right-hand edge of the display region assumed a variety of shapes, and the other three edges were straight (see Figure 1). In the move condition, a sparse regular array of nine points moved toward the upper right-hand corner of the display region at a uniform velocity $\left(9.6^{\circ} \mathrm{per}\right.$ second during the experiment, see Figure 1, upper panel). The computer extinguished a point only as it "impacted" either the right-hand or the upper edge of the display region. The regular spacing of the points coupled with their constant velocity produced impacts on these two edges that were evenly distributed in time and space. Extinguished points were "wrapped around" the display region so that points were being continuously propagated at either the left-hand or lower edges of the region. The sparseness of dots in the array was such that if the array was not moving, the edges of the display region were completely invisible (Figure 1, upper panel).

All trials in the flash condition were the same as their counterparts in the move condition, except for one difference: the moving-point array was invisible as it swept across the display region. The points became visible for one cycle of the computer program $(20 \mathrm{msec})$ only as they impacted either the right-hand or upper edge of the display region. 


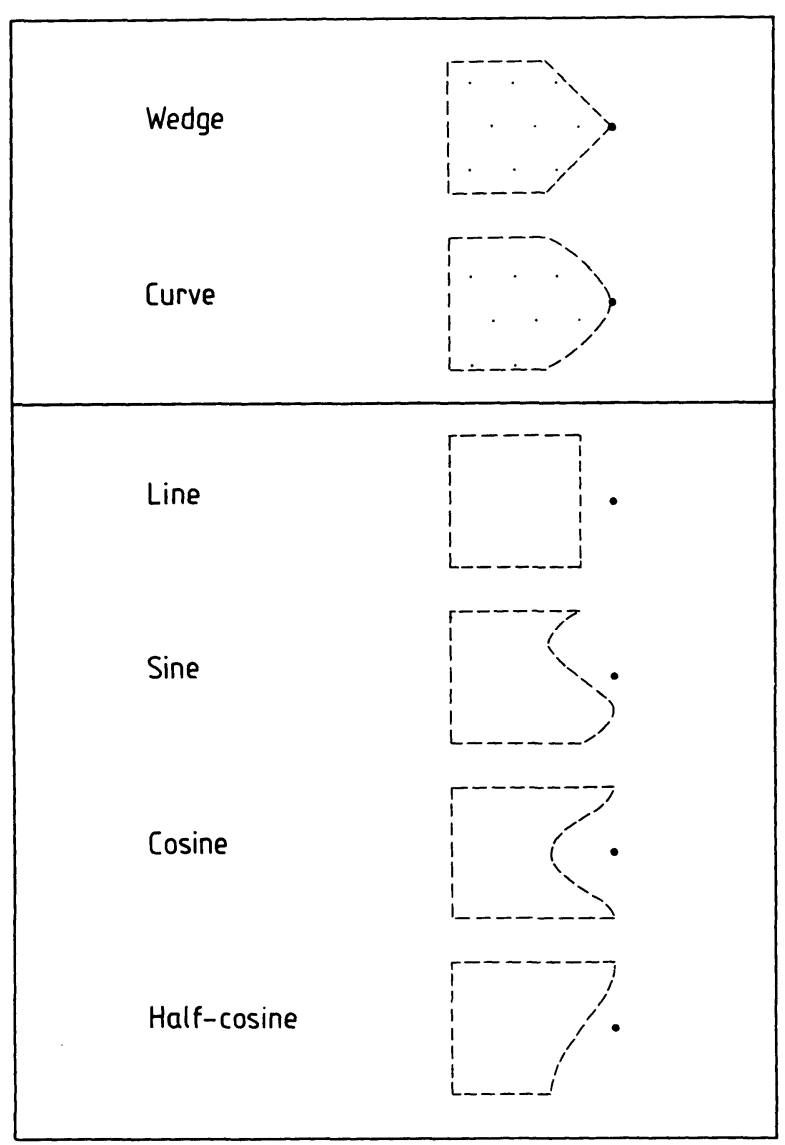

Figure 1. Stimuli used in the demonstrations and experiment. The broken lines indicate the perimeters of the display regions; the curves of the six right-hand edges are sine/cosine functions. The dot to the right of each display region indicates the position of the fixation point. In the upper panel, the points within the two regions indicate the moving array used in the move condition.

Thus the points were flashed on the CRT screen at locations corresponding precisely to either the upper or right-hand edges, in exactly the same spatiotemporal sequence as the extinction of the moving points in the move condition.

\section{Subjects and Procedure}

There were 8 observers ranging in age from 18 to 20 years. These subjects received course credit for their participation in the experiment. They had corrected visual acuity of at least 20/25 and were naive about the goal of the experiment.

Initially, as a demonstration of the illusion to observers, we varied both the shape of the right-hand edge of the display region, using each of the shapes in Figure 1, and the density of the point array (up to 25 points). We also varied the uniform velocity at which the points traveled. The observers reported their perceptions to the experimenter under both move and flash conditions.

In the actual experiment, the move and flash conditions were presented in random order, and each condition consisted of $\mathbf{3 0}$ trials, again randomly ordered. Twelve of these trials presented the wedge right-hand edge, whereas 12 presented the curve edge (Figure 1, upper panel). The other six trials were "catch" trials, which consisted of one of the other four edges (Figure 1, lower panel). The subject started a trial in his/her own time by depressing a microswitch and staring at the fixation spot. In the move condition, the nine points remained stationary within the display region for $3 \mathrm{sec}$, then moved toward the upper right. The time of movement was $200 \mathrm{msec}$ for half the trials and $300 \mathrm{msec}$ for the other half. After the timed movement, the screen blanked. These display times allowed either five or seven points, respectively, to impact the righthand edge. Short display times were used to nullify the possible effects of eye movements on edge localization. From trial to trial, the direction of motion of the points was randomly varied by a few degrees, so that the points would never disappear at the same locations for a given edge and display time. The trials in the flash condition corresponded precisely to their move condition counterparts, except that the points were visible only as they impacted the upper or right-hand edge. The subject's task was to say whether the right-hand edge was the wedge, the curve, or other. Before the experimental trials, subjects practiced viewing the display for both conditions under a variety of display times and right-hand edges.

\section{RESULTS AND DISCUSSION}

During the demonstrations, all observers reported that the move condition created a compelling subjective contour that circumscribed the display region and corresponded to one of the shapes in Figure 1. They described the contour in terms of the attributes of the classic illusion of Kanisza (1976; for review, see Parks, 1984). First, the occluding form, or the area outside the display region, seemed darker than the display region itself. Second, the occluding contour seemed to be part of a "window" that was interposed between the observer and the moving display. Finally, the subjective contours appeared sharp and of distinct shape.

The subjective contours were visible in the move condition with arrays as sparse as nine points. For a given velocity, increasing the number of points increased the strength of the contour, but this also had the effect of increasing the suggestion of an occluding form when the display was static. In general, slowing the velocity of the array destroyed the percept of a contour around the display region. The formation of a subjective edge required at least six impacts per second spaced along the "edge's" length. However, if the moving array of dots suddenly stopped, the subjective contour "faded" over a period of a second or so, in a manner similar to the way it took time to appear.

On the other hand, no occluding subjective form was seen in the flash condition. Rather, the points seemed to "jump" randomly along the edges in a manner similar to phi movement. However, all subjects agreed that the delineation of an edge was more cogent in the flash condition than in the concomitant move condition. These impressions were confirmed by data from the experiment. Trials were scored according to whether subjects correctly reported the shape of the right-hand occluding edge as wedge, curve, or other. Figure 2 shows the averaged data for the two display times, pooled across the wedge and curve trials. The flash condition produced significantly better identification of the shape of the right-hand edge than did the move condition $[F(1,7)=23.84, p<.005]$. In fact, the interaction between display time and presentation condition almost reached significance $[F(1,7)=$ $4.95, p=.06$ ], suggesting that more information for edge localization might have actually decreased performance in the move condition. It follows that the clearly visible 


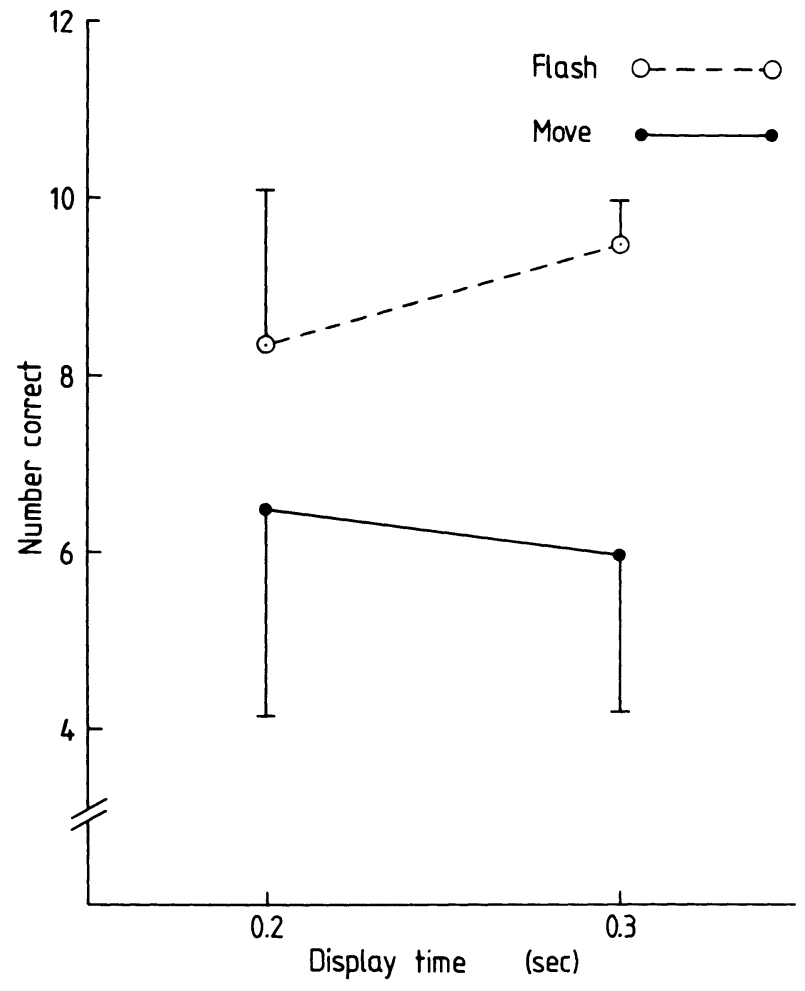

Figure 2. Averaged data for the two display times used in the move and flash conditions of the experiment. Error bars are one standard deviation.

paths of the moving points constituted noise for the visual system, which was required to store the relative locations of the points' extinction as precisely as possible.

Prazdny (1985) recently claimed that the inducing elements of subjective contours must be defined in the brightness domain. In fact, earlier researchers (Brigner \& Gallagher, 1974; Frisby \& Clatworthy, 1975) had taken a stronger position: the illusory forms were simply a special case of simultaneous brightness contrast. On the other hand, the principal conclusion of this research note, as well as that of Prazdny (1986), is that a classic subjective contour can be formed without the existence of an inducing element that is defined by brightness. ${ }^{1}$ Instead, the contour is formed if and only if there is both spatial and temporal interpolation, and the exact nature of this process requires further investigation.

\section{REFERENCES}

Bradley, D. R., Lee, K. (1982). Animated subjective contours. Perception \& Psychophysics, 32, 393-395.

BridgemAN, B., \& MAYER, M. (1983). Failure to integrate visual information from successive fixations. Bulletin of the Psychonomic Society, 21, 285-286.

Brigner, W. L., \& Gallagher, M. B. (1974). Subjective contour: Apparent depth or simultaneous brightness contrast? Perceptual \& Motor Skills, 38, 1047-1053.

Coren, S. (1972). Subjective contours and apparent depth. Psychological Review, 79, 359-367.

Frisby, J. P., \& Clatworthy, J. L. (1975). Illusory contours: Curious cases of simultaneous brightness contrast? Perception, 4, 349-357.

KaniszA, G. (1976). Subjective contours. Scientific American, 234(4), 48-52.

Kaplan G. A. (1969). Kinetic disruption of optical texture: The perception of depth at an edge. Perception \& Psychophysics, 6, 193-198.

Kellman, P. J., \& Cohen, M. H. (1984). Kinetic subjective contours. Perception \& Psychophysics, 35, 237-244.

KlymenKo, V., \& Weisstein, N. (1981). The motion-induced contour. Perception, 10, 627-636.

PARKs, T. E. (1984). Illusory figures: A (mostly) atheoretical review. Psychological Bulletin, 95, 282-300.

Petersik, J. T., \& McDill, M. (1981). A new bistable motion illusion based upon 'kinetic optical occlusion.' Perception, 10, 563-572.

Prazdny, K. (1985). On the nature of inducing forms generating perceptions of illusory contours. Perception \& Psychophysics, 37, 237-242.

PrazDNy, K. (1986). Illusory contours from inducers defined solely by spatiotemporal correlations. Perception \& Psychophysics, 39, 175-178.

\section{NOTE}

1. In another context, Petersik and McDill (1981) produced a "shadowy" subjective form-that is, one without well-defined edgespurely by temporal changes in a square-wave luminance pattern.

(Manuscript received for publication October 24, 1986.) 